\title{
Weld Distortion Control Methods and Applications of Weld Modeling
}

\author{
F. W. Brust
}

Paul Scott

\begin{abstract}
A virtual fabrication technology (VFT) modeling procedure is introduced in this paper. It is a state-of-theart fabrication modeling tool which can handle all aspects of fabrication including forming, cutting, machining, and welding. This simulation tool was developed by Battelle and Caterpillar over the past ten years. It can model extremely large and complicated structural fabrications. Extensive full-scale experiments have validated the accuracy and predictive power. It can be used to reduce fabrication cost and improve quality by minimizing and controlling distortions. Several application examples are presented to illustrate how to apply this tool in welded structure design and manufacture.
\end{abstract}

\section{INTRODUCTION}

Many researchers have tried to develop modeling methodologies to simulate the welding process, but the modeling techniques that have been developed have often been too complex, less accurate, or too labor intensive to be applied industrially. Although significant progress has been made in the finite-element modeling of welding processes in resent years, many of the modeling techniques are still far short of being used successfully for the control of residual stress and distortion in actual structures.

Since 1996, Battelle and Caterpillar have been working together to develop an industrial-use methodology and user-friendly software for predicting weld residual stress and distortion in large and complicated structures. Brust et al (1981, 1998, 2003), Dong and Brust (2000) and Yang et al (2000a-b, 2002a-c) performed residual stress analyses for actual large structures. A large amount of manpower and equipment was invested for model development, program coding, and validation. Finally, a Virtual Fabrication Technology (VFT) and a weld modeling computer tool was developed and is now used routinely in the Caterpillar design process worldwide. VFT is a state-of-the-art fabrication simulation technology that allows rapid solutions for large, complex metallic structures containing both single-pass and multi-pass welds and allows the user to consider or input all critical variables. It can be used in product design stages to help the weld design and in the manufacture stage to determine the optimal weld processes to minimize welding-induced distortion.

\section{VIRTUAL FABRICATION TECHNOLOGY MODEL}

The procedure of simulating the arc welding processes with the VFT is discussed in Brust et al (1998, 2000, and 2005). Three kinds of modeling procedures: local weld residual stress analysis procedure, moving-arc analysis procedure, and lump-pass analysis procedure are included in the software. Local weld residual analysis procedure has been widely used in predicting and mitigating weld residual stress in the pressure vessels (Brust, 2005, and references sited therein) and nuclear piping systems. The moving-arc analysis procedure was developed for predicting distortion in large and complicated welded structures. 


\section{DISTORTION CONTROL TECHNIQUES AND ADVANTAGES}

Two tremendous advantages are obtained by developing fabrication solutions via the computer. First, designing the fabrication to minimize or control distortions can significantly reduce fabrication costs. Second, controlling the fabrication-induced residual stress state can significantly enhance the structure's service life. For distortion control, fabrication design via modeling can:

- eliminate the need for expensive distortion corrections

- reduce machining requirements

- minimize capital equipment costs

- improve quality

- permit pre-machining concepts to be used

Residual stress control via modeling can:

- Reduce weight

- Maximize fatigue performance

- Lead to quality enhancements

- Minimize costly service problems

- Improve damage resistance during attack (e.g., Naval structures)

Fabrication modeling tools were specifically developed for this purpose. Weld process models are sophisticated physics/mathematics based computer modeling software tools for use in optimizing and designing metal fabrication processes for industry. Savings from using a weld process model can be significant often exceeding millions of dollars per product.

There are a number of methods that can be used to control weld induced distortions, residual stresses, or both. Which method is practical for a given product depends on many factors including cost. Some of the more popular methods for controlling welds include weld sequencing, pre-cambering, thermal tensioning, heat sink welding, and fixture design, among many others. It is important to note that fabrication modeling tools can be used to develop new control methods since the new methods can be first attempted on the computer. Some of these methods will be considered in the examples discussed later.

\section{Factors Affecting Fabrication}

Welding, along with thermal cutting, bending and machining, is usually referred as the key manufacturing "building block". Most fabricated structures start with steel plates received from a steel supplier. The plates are cleaned, typically by shot blasting or by using chemical means. Thermal cutting processes, such as oxy-fuel flame cutting, plasma cutting, and recently laser cutting, are used to cut the plate into desired profiles and dimensions. Bending or forming process could be used to change the parts into the desired shapes. The piece parts are then tacked together in a fixture and welded into an assembly. Machining may be used at either part or assembly level to achieve the tolerance of critical dimensions.

Each operation introduces manufacturing variability. The final quality of the assembly depends on the quality control of each process. In addition, the quality of one process could be 
affected by the upstream processes and will influence the downstream processes. Process simulations have been reported extensively to understand the physics and science of manufacturing processes as illustrated in Figure 1. The following is a brief description of the thru-process simulations associated with each manufacturing building block.

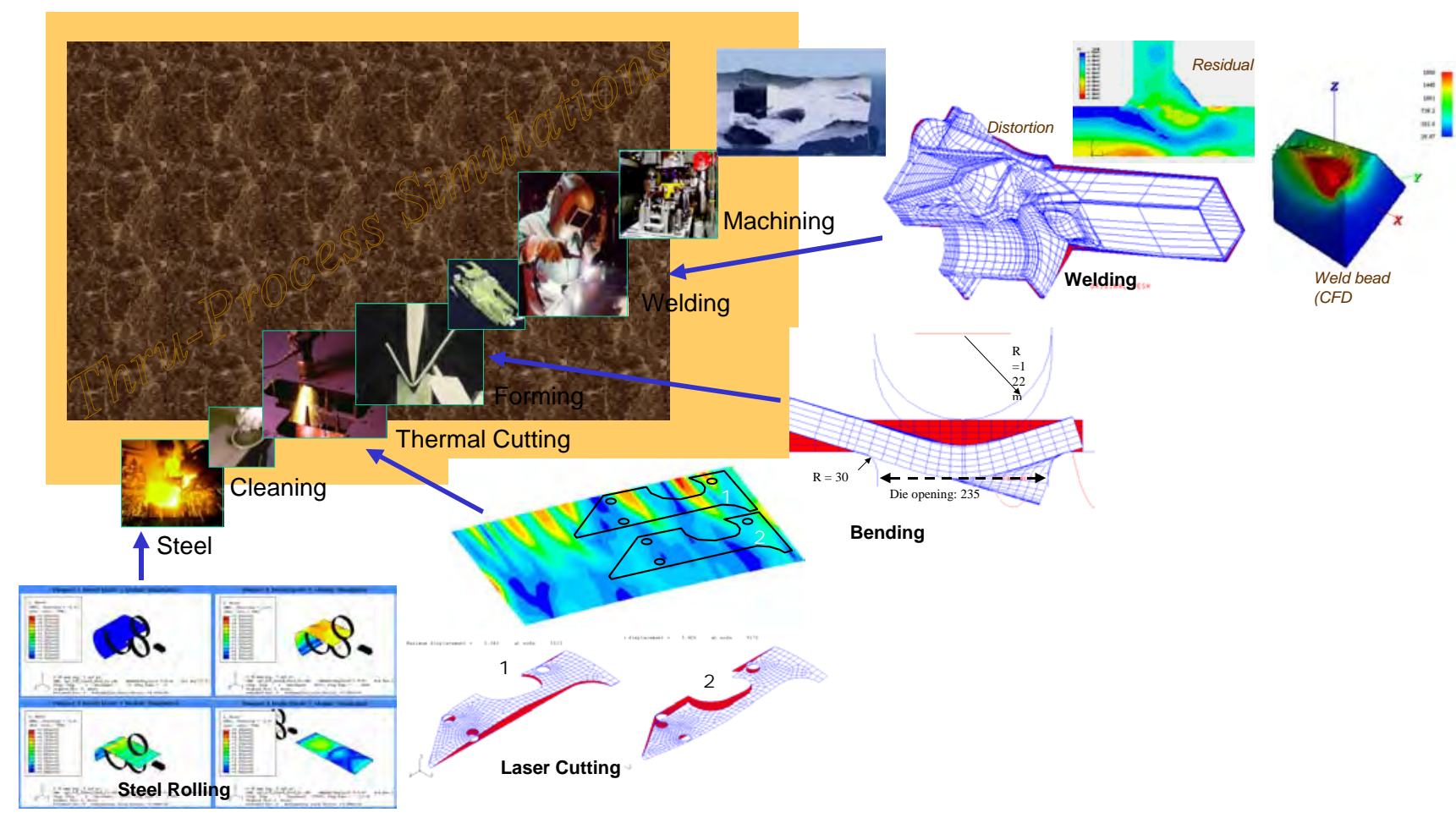

Figure 1. Processes involved in metal fabrication.

Steel rolling simulation - The model simulates the typical steel plate making processes focusing on the locked-in residual stresses. Such residual stresses could alter the plate response during the cutting process. Therefore, the residual stress produced during rolling is one of the key inputs to the downstream thermal cutting simulation.

Shot blasting simulation - The model predicts the results of shot impact on the plate surface. It simulates the plastic deformation, residual stresses and oxide removal.

Thermal cutting simulation - The model simulates the distortion and residual stresses that result from thermal cutting processes. It predicts the thermal mechanical response of the cutting process as well as the interaction of thermal induced distortion with the residual stresses from steel making process. The simulation can improve the cutting accuracy by providing critical information that is used to optimize the part nesting and cutting sequence.

Bending simulation - The model simulates the in process mechanical response of the material. The simulation predicts the bending accuracy and spring back based on the material variables and process parameters.

Welding simulation - The model simulates wide range of welding phenomena including thermodynamic of weld pool, metallurgical transformations in the weld and its heat-affected zone and thermal 
mechanical response of the structure during the welding process. Simulation provides a unique tool for welding engineer to design and optimize the welding process.

\section{GENERAL EXAMPLES}

Bi-metallic Nuclear Pipe Weld and Crack Growth Study. As a result of recent unexpected cracking in the hot leg bi-metallic welds in some pressurized water reactors (PWR), the US Nuclear Regulatory Commission (NRC) asked Battelle to investigate the issue. This example illustrates how the fabrication modeling system may be used not only to model the full weld fabrication process, but to add service loads for an in depth study of the problem.

Figure 2a shows schematically the system of interest. The nozzle from the A508 pressure vessel is welded to a stainless steel pipe using an inconel buttering process (on the A508) first, followed by the inconel weld (the ferritic steel is also clad with a stainless steel). The pipes are large diameter $(760 \mathrm{~mm})$ and thick $(63 \mathrm{~mm})$. The issue of concern was whether circumferential cracks (which can lead to

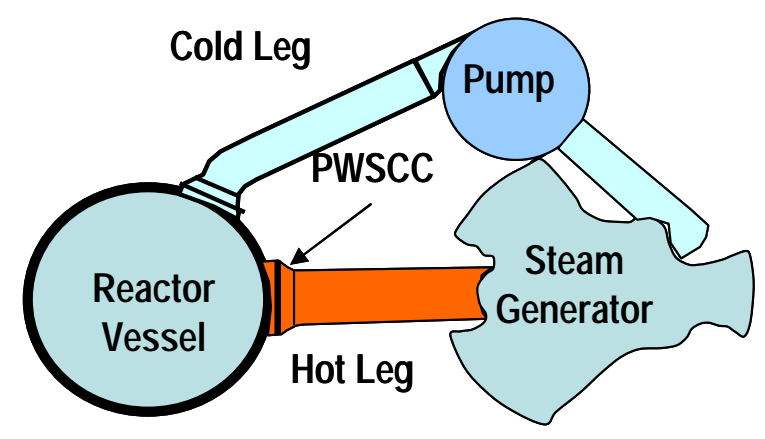

Figure 2a. Hot Leg Nuclear Crack



(615 F Normal Operating Loads)

Figure 2b. Hot Leg Nuclear Crack Problem

catastrophic coolant loss) can grow faster than axial cracks (which can be tolerated temporarily) and whether similar problems might exist in the numerous other worldwide PWR nuclear plants. The problem was further complicated by the fact that the weld of concern had already undergone weld repairs.

The entire weld process was first modeled. The analysis consisted of (1) preheat and modeling buttering of the nozzle, (2) post weld heat treat simulation of the buttered nozzle, (3) preheat and welding of the nozzle to stainless steel pipe, (4) grind out of a portion of the weld (5) repair weld analysis (6) hydro-test analysis (7) service load heat up (8) service load application, (9) introduction of cracks and determination of stress intensity factors (using a finite element alternating technique), and (10) SCC crack growth analysis. It was critical to include the entire history of stress, plastic and creep strain throughout the analysis since service stresses depend on this history. The final residual stress state at operating temperature and loads is shown in Figure 2b.

The scale ranges from $50 \mathrm{ksi}$ (345 Mpa - red) to -50 ksi (345 Mpa - purple). The axial stresses are clearly lower than hoop stresses, and the large hoop stresses are mainly confined to the inconel weld. Stress intensity factors were then calculated for about 100 elliptical cracks inserted axially and circumferentially into this stress state (using FEAM, (FRAC@ALT, 2003)) and subsequent three-dimensional SCC analysis 
was performed (see Figure $2 \mathrm{~b}$ ). The results indicated that axial cracks should grow and break through the pipe wall about twice as fast as circumferential cracks. This prediction correlated with crack experience to date. Moreover, the axial cracks should be confined to the inconel butter and weld zone.

Circumferential cracks may grow to be long (circumferentially) before breaking through the pipe wall. This suggests that loss of coolant due to a double-ended pipe break is not likely. This clearly illustrates a case where the use of a model can be used to quantify and correct a service cracking problem. Many more examples of this are included in the references sited here and references therein.

Pre-straining Technique for Distortion Control. Pre-staining is another technique for control welding-induced distortion in heavy industries. With pre-straining, some plates are pre-bent, plastically, before welding. This is in contrast to pre-cambering, where the bending during the weld process is elastic. This may have applications in many large ship fabrication areas where thick plate must be welded and distortions controlled, such as bulkheads.

Before assembling welded structure, plates are bent into permanent shape based on predictions using VFT. After welding, welding-induced distortion makes the bent shape become straight. To determine the pre-bent shape and magnitudes, a large amount of experiments needed to be conducted (in the past) before weld modeling software was available.

In Fig. 3a and Fig. 3b, the pre-straining technique is described to mitigate distortion resulting from the J-groove circumferential fillet weld between a very long cylinder to a plate. Before welding, the plate was bent, as shown in Fig. $3 \mathrm{a}$ and $3 \mathrm{~b}$. The end edge of the plate was pre-bent to about $7 \mathrm{~mm}$ away from middle straight part of the plate in the cylinder axle direction, as marked in Fig. 3a. It is import to note that the pre-bent plate has 3 nearly straight sections with two bend radii. After welding, the pre-bent plate becomes almost flat in Fig. $3 b$.

It should be pointed out that the plate was only bent in one dimension. This is because the plate is

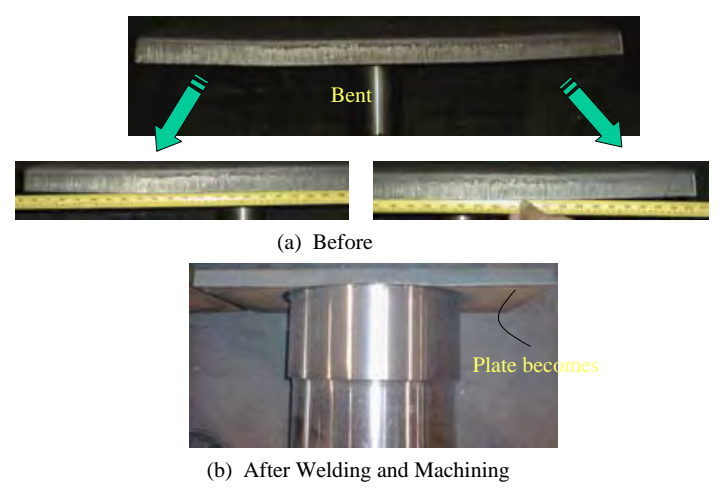

Fig. 3a Shop Floor Practice of Pre-Straining

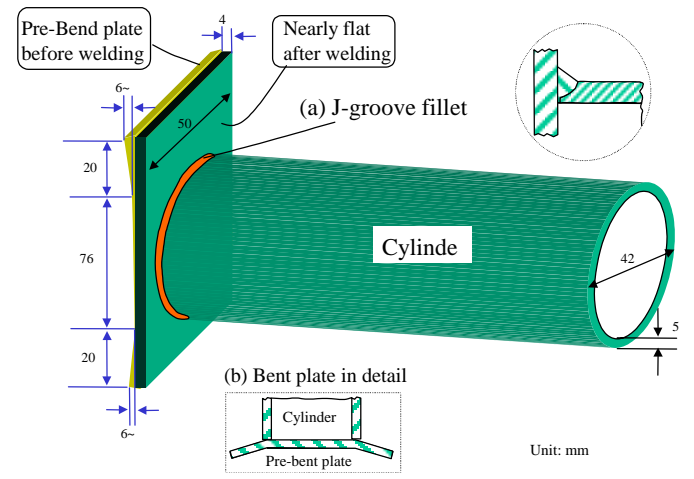

Fig. 3b Pre-Straining Technique for Mitigating (Dimensions in cm)

rectangular and the J-groove circumferential fillet weld is close to the two edges of the short side of the rectangular. If the plate is square and the center of circumferential weld is around the center of the plate, it is necessary to bend the plate in two dimensions. This pre-straining technique is easy to use and very effective. The use of weld modeling software makes the design of this type of procedure practical for any type of large structure.

Ship Panel Construction. The structure illustrated in Figure 4 is part of the hull of a US Navy destroyer, fabricated by a US ship fabricator. This very large section incorporates numerous tee fillet welds, both vertical and horizontal. The key point illustrated here is the manpower effort required to perform it this analysis. A solution to the distortion control of a panel such as this would mainly be sequencing. This analysis was performed quickly. 
- $\quad$ Number of welds modeled $=154$.

- $\quad$ Model generation: person 1, 8 hours and person 2, 24 hours.

- $\quad$ Total shell elements 30,000.

- $\quad$ Set up thermal analysis, person 1, 8 hours.

- $\quad$ Set up stress analysis, person 2, 8 hours.

- $\quad$ Computer running time (three days - depends on computer - SUN Workstation here). This would be much faster today on an Itanium chip machine.

- $\quad$ Results analysis and summary: person 1, 16 hours and person 2, 20 hours.

- $\quad$ Total human hours: person 1, 32 hours; person 2, 52 hours.

- $\quad$ Modeling procedures: lump-pass modeling procedures.

Since the structure had single pass welds, a relatively simple shell model was used and only one demonstration analysis was performed. Typically, additional analyses using different sequences, precambering, or other distortion control technique, would be required, but once the baseline model is built, setting up additional analyses is trivial.

An illustration of the distortion control procedures for typical ship-type fabrications are found in Figure 4. As shown in the diagram incorporated in Figure 4, they were joined using double sided welds where the weld was first finished along one side and then the other side was completed. After the predictions were made, predicted results were compared with service welded panels (qualitatively). The distortion predictions match qualitatively with those found after actual welding. The actual distortions were not measured exactly. The 'bow' distortion was predicted to be about 1.75 -inches $(44.5 \mathrm{~mm})$.

More importantly, it is clear that weld parameters (e.g. heat input, torch speed, material) strongly affect the final distortion. The $40^{\prime}$ by $80^{\prime}(12 \mathrm{~m}$ by $24 \mathrm{~m})$ hull assemblage of Figure 4 was modeled for illustration using a typical welding sequence applied to $6 \mathrm{~mm}$ thick plate. The resulting distortions of the full structure illustrate the issues of concern, showing by comparison with the initial (un-deformed) shape that the hull assemblage bows up along the long length. An assemblage like that in Figure 4, for example, will require five to ten separate analyses with varying parameters before the optimum sequence for distortion control can be realized. Again, weld sequencing should be the preferred control method.

\section{Submarine Hull Manufacture}

Consider a $2.5 \mathrm{~m}$ diameter and $2.3 \mathrm{~m}$ long cylinder welded to a $101.4 \mathrm{~mm}$ thick ring with the GMAW welding process. Both cylinder and rings are made of high strength steel. The cylinder is originally a flat plate, edge prepared according to specified weld joint type, and then rolled, and welded longitudinally with a multi-pass single bevel seam weld. The cylinder was then welded to the end ring with a double side 
half-inch Tee fillet weld. This might be considered to be part of a submarine hull and ring stiffener weld problem.

Three sequences were analyzed. The first sequence is to tack the cylinder, perform the multi-pass seam weld, and then weld the cylinder to the end ring with the outside fillet, and inside fillet. It was found that the cylinder was significantly distorted in the radial direction a maximum value of about $15 \mathrm{~mm}$. The second sequence is to tack the cylinder, then tack the cylinder to the end ring from outside and inside Tee fillet locations, perform seam weld, and weld outside the Tee fillet and inside the Tee fillet. It was found that the radial direction distortion was significantly reduced but the roundness of the cylinder was still not within the design requirement. The third sequence has the same tacking procedures as the sequence two. But at first the inside Tee fillet was welded, then outside Tee fillet, and finally the seam weld. It was found that the radial distortion is reduced less than $1 \mathrm{~mm}$ and can meet the design requirement. The reason that the third sequence produces less distortion is that after the Tee fillet weld the cylinder is stiffer so that the seam weld induced distortion is smaller. Sequencing is clearly the preferred procedure for this case. Many additional analyses were performed, including residual stresses. These are not shown here and more details can be seen in Brust 2005.

\section{CONCLUSION}

Welding-induced distortion control techniques, weld design optimization, pre-straining, optimum welding sequence and pre-cambering, were investigated using fabrication modeling software based on several industrial products and ship type components. The potential to design flame straightening schemes was also discussed. The power of computational modeling should be clear. By using such software (VFT here but other software exists as well) the optimum distortion control parameters could be obtained from the computer simulation.

\section{REFERENCES}

Brust, F.W., Dong, P., Zhang, J., Cao, Z, Yang, Y. P., and Hong, J.K., "Weld Process Modeling and It's Importance in a Manufacturing Environment", Proc. 1998 Earthmoving Industry Conference and Exposition, Peoria, Ill, April 8-9, 1998, SAE Technical Paper Series \#981510.

Brust, F. W., Kim, D. S., "Mitigating Welding Residual Stress and Distortion”, Chapter 8, in Processes and Mechanisms of Welding Residual Stress And Distortion, Ed. Z. Feng, Woodhead Publishing Limited, Cambridge, UK, 2005.

Chen, L., Zhang, Z., and Brust, F. W.., "Modeling Distortion and Residual Stress During Welding: Practical Applications", Chapter 7, in Processes and Mechanisms of Welding Residual Stress And Distortion, Ed. Z. Feng, Woodhead Publishing Limited, Cambridge, UK, 2005.

Dong, P., and Brust, F.W., "Welding Residual Stress and Effects to Fracture in Pressure Vessel and Piping Components: A Millennium Review and Beyond," Journal of Pressure Vessel Technology, 2000, 122: pp. 329-328.

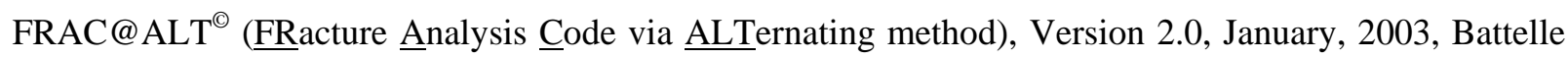
Memorial Institute.

Yang, Y. P., Chen, X. L., Dong, P., Varol, I. Cao, Z., and Hong, J. K., "Experimental and Finite element Study of Laser Cutting Induced Distortion in a Production Environment," International Conference on Computer Engineering and Science, 31 July-2 August, 2002, Reno, NV, USA. 